\title{
Cosmological bounds on dark-matter-neutrino interactions
}

\author{
Gianpiero Mangano, ${ }^{1}$ Alessandro Melchiorri, ${ }^{2}$ Paolo Serra, ${ }^{2}$ Asantha Cooray, ${ }^{3}$ and Marc Kamionkowski ${ }^{4}$ \\ ${ }^{1}$ Physics Department and Sezione INFN, University of Naples "Federico II", Via Cintia, 80126 Naples, Italy \\ ${ }^{2}$ Physics Department and Sezione INFN, University of Rome "La Sapienza", P.le Aldo Moro 2, 00185 Rome, Italy \\ ${ }^{3}$ Center for Cosmology, Department of Physics and Astronomy, 4129 Frederick Reines Hall, University of California, \\ Irvine, California 92697, USA \\ ${ }^{4}$ California Institute of Technology, Mail Code 130-33, Pasadena, California 91125, USA
}

(Received 13 June 2006; published 15 August 2006)

\begin{abstract}
We investigate the cosmological effects of a neutrino interaction with cold dark-matter. We postulate a neutrino that interacts with a "neutrino-interacting dark-matter" (NIDM) particle with an elasticscattering cross section that either decreases with temperature as $T^{2}$ or remains constant with temperature. The neutrino-dark-matter interaction results in a neutrino-dark-matter fluid with pressure, and this pressure results in diffusion-damped oscillations in the matter power spectrum, analogous to the acoustic oscillations in the baryon-photon fluid. We discuss the bounds from the Sloan Digital Sky Survey on the NIDM opacity (ratio of cross section to NIDM-particle mass) and compare with the constraint from observation of neutrinos from supernova 1987A. If only a fraction of the dark matter interacts with neutrinos, then NIDM oscillations may affect current cosmological constraints from measurements of galaxy clustering. We discuss how detection of NIDM oscillations would suggest a particle-antiparticle asymmetry in the dark-matter sector.
\end{abstract}

DOI: 10.1103/PhysRevD.74.043517

PACS numbers: $98.80 . \mathrm{Cq}$

\section{INTRODUCTION}

Flat cosmological models with baryons (about $5 \%$ of the total energy content of the Universe), cold dark matter (CDM, 25\%), cosmological constant (or dark energy, $70 \%$ ), and an adiabatic, nearly scale-invariant spectrum of density fluctuations explain most cosmological observations. However, we still lack a satisfactory understanding of both dark matter and dark energy, a puzzle for both particle physics and cosmology.

The most favored candidates for dark matter are cold, collisionless massive particles, which are nonrelativistic for most of the history of the Universe and so can cluster gravitationally during matter domination. Candidates for these dark-matter particles can be found in supersymmetric extensions of the standard electroweak model-namely neutralinos with mass on the order of $100 \mathrm{GeV}$ [1] - or in other theories (e.g., the axion, which may arise in the Peccei-Quinn mechanism [2]). These cold-dark-matter models account well for cosmic microwave background (CMB) observations on the largest scales as well as measurements of the large-scale distribution of galaxies.

However, observations on galactic and subgalactic scales may conflict with the predictions, from numerical simulations and analytic calculations, of CDM models. Indeed, cold and collisionless dark-matter models seem to predict an excess of small-scale structures [3], and numerical simulations [4] predict far more satellite galaxies in the Milky Way halo than are observed.

Several solutions have been proposed to explain these discrepancies, for example, inflationary models with broken scale invariance [5]. However, most other explanations invoke modifications to the properties of dark-matter particles. For example, a warm-dark-matter candidate, like a sterile neutrino, has been suggested because it suffers free streaming and suppresses the matter power spectrum on small scales [6]. A dark-matter particle that results from decay of a short-lived charged particle can also suppress small-scale power [7]. Other possibilities include a darkmatter particle that interacts with other particles such as photons [8-10], neutrinos [11,12], or self-interacting dark matter [13]. For a review of different alternative scenarios to standard collisionless cold dark matter, see Ref. [14].

In this paper, we investigate the possibility of a neutrinointeracting dark-matter (NIDM) component. If dark matter and neutrinos interact, there was an epoch in the very early Universe in which they were strongly coupled. Darkmatter perturbations that entered the horizon during this period would then be erased because of diffusion damping, and the suppression scale will depend on the dark-matterneutrino interaction. Even if only a fraction of the dark matter interacts with neutrinos, a pattern of oscillations in the matter power spectrum arises, much like the oscillations in the baryon-photon fluid.

In the following, we limit our study of DM-neutrino couplings to effects on cosmological scales in the frequency range smaller than $k<0.2 h \mathrm{Mpc}^{-1}$-i.e., on scales where linear perturbation theory is viable. We consider flat cosmological models with an adiabatic and nearly scale-invariant spectrum $P(k) \sim k^{n}$ of density perturbations where $n=0.97$. Unless explicitly stated, the energy content of the Universe corresponds to the standard $\Lambda \mathrm{CDM}$ model with baryons contributing as $\Omega_{b}=0.05$ and a colddark-matter energy density $\Omega_{\mathrm{dm}}=0.25$. We also choose a Hubble parameter $h=0.73$, a standard value 3.04 for the effective number of (massless) neutrinos [15], and dark energy in the form of a cosmological constant with 
equation-of-state parameter $w=-1$. The interaction between dark matter and neutrinos is given in terms of the opacity $Q=\left\langle\sigma_{\mathrm{dm}-\nu}|v|\right\rangle / m_{\mathrm{dm}}$, the ratio of the thermal averaged dark-matter-neutrino cross section to the mass of the dark-matter particle.

The paper is organized as follows. In the next section, we discuss a class of models of neutrino-dark-matter interaction for both scalar and spinor dark-matter candidates and obtain an estimate for the opacity $Q$. In Sec. III, we outline the cosmological consequences of a NIDM component, and we compare those predictions with the latest data on galaxy clustering from the Sloan Digital Sky Survey (SDSS). In Sec. IV, we consider astrophysical constraints, particularly those from observation of neutrinos from supernova 1987A. Finally, in the last section, we report our conclusions.

\section{THE NEUTRINO-DARK-MATTER INTERACTION}

The possibility of new neutrino interactions with exotic matter fields and their cosmological implications have been recently considered by many authors. Couplings with a light scalar or pseudoscalar boson, as in the Majoron model [16-19], can have sizeable effects on the $\mathrm{CMB}$ and the power spectrum of large-scale structure (LSS), and might lead to a neutrinoless universe for a massless or very light scalar field [19].

A different class of interactions of neutrinos with DM, and more generally of DM with the electromagnetic plasma as well, was put forward in a series of papers discussing the possibility that DM consists of particles with mass $m_{\mathrm{dm}}$ in the $\mathrm{MeV}$ range [20-24]. If the relic abundance of these particles is produced via the freeze-out of annihilation processes and yet corresponds to the observed DM energy density today, they must interact with stronger than weak interactions, since the cross section for annihilations via the exchange of a massive particle as, e.g., a vector boson, typically decreases at the freeze-out as the square of the DM-particle mass.

The implications of this scenario for big bang nucleosynthesis (BBN) have been considered in Ref. [25]. With DM-neutrino interactions producing the required DM annihilation cross section of a few pico-barns (for an $s$-wave annihilation) at decoupling, the mass range $m_{\mathrm{dm}} \leq$ $10 \mathrm{MeV}$ is disfavored. In fact, tightly coupled DM particles give in this case a non-negligible contribution to the total energy density, and the neutrino-photon temperature ratio is increased because of the entropy release from DM to neutrinos. Both effects conspire to produce an order one extra effective neutrino species and thus an increased primordial helium abundance, which is difficult to reconcile with the present determination of the ${ }^{4} \mathrm{He}$ mass fraction; see, e.g., Refs. [26-29].

Neutrino coupling to DM particles with mass in the $\mathrm{MeV}$ range can also be bound using neutrino fluxes from type II supernovae [30], such as SN1987A, again resulting in a constraint $m_{\mathrm{dm}} \geq 10 \mathrm{MeV}$. It is worth observing however, that this bound only applies if these particles have large elastic-scattering cross sections with nucleons, larger than neutrino-nucleon ones, whose effect is to shift outward the neutrinosphere and thus leads to a lower neutrino decoupling temperature.

In the following, we consider a DM-neutrino coupling even stronger than those considered in Refs. [20-24], so that the resulting large scattering cross sections might lead to detectable effects in the LSS power spectrum. In fact, as already emphasized in Ref. [21], a DM-neutrino coupling which leads to an order pico-barn annihilation cross section can only affect the power spectrum on very small scales, since scattering processes freeze out quite early in time. In this case, LSS forms as in the presence of the usual collisionless DM fluid, while neutrinos act via free streaming and suppress power on scales smaller than the acoustic horizon at the time they become nonrelativistic.

We assume $m_{\mathrm{dm}} \geq 10 \mathrm{MeV}$, since our large DMneutrino coupling implies that the bound obtained in Ref. [25] applies a fortiori. Notice that our working hypothesis potentially leads to the problem of how a sizeable relic abundance of a DM particle actually forms, since a stronger coupling with neutrinos enhances their annihilation rate and leads to negligible relic abundance today. We will comment on this point later and emphasize that our scenario requires an asymmetry between the DM particle and antiparticle produced at an early stage.

The interaction Lagrangian density depends upon the spin content of the DM field, denoted in the following by $\psi$. We consider here the case of a nonself-conjugated scalar particle or a Dirac spinor and interaction terms that admit a conserved global $U(1)$ charge with $\psi$ transforming with a phase under the corresponding transformations. For a scalar $\psi$ we can write

$$
\mathcal{L}_{\text {int }}=h \bar{F}_{R} \nu_{L} \psi+\text { H.c., }
$$

where $F$ is a spinor field. Similarly, DM coupling to neutrinos can be also introduced via the interaction with an intermediate vector-boson field $U_{\mu}$,

$$
\begin{aligned}
\mathcal{L}_{\text {int }}= & i g_{\psi}\left(\psi^{*} \partial^{\mu} \psi-\psi \partial^{\mu} \psi^{*}\right) U_{\mu}+g_{\psi}^{2} \psi^{*} \psi U_{\mu} U^{\mu} \\
& +g_{\nu} \bar{\nu}_{L} \gamma^{\mu} \nu_{L} U_{\mu} .
\end{aligned}
$$

In both cases, we consider the $F$ or $U$ field to have masses of order of $\mathrm{MeV}$ or larger to forbid $\psi$ decays at tree level, which would erase any relic abundance of $\psi$ unless the couplings are tuned to be very small. In a way, this assumption is far from being ad hoc for the case of a coupling as in Eq. (1). If the $F$ field is lighter than $\psi$, we simply have to shift our perspective and consider $F$ as a candidate for light dark matter rather than the $\psi$ field.

In the range of neutrino temperature $T \leq \mathrm{MeV}$ we are interested in, the thermally averaged $\psi$-neutrino scattering 
cross section is

$$
\left\langle\sigma_{\mathrm{dm}-\nu}|v|\right\rangle \sim|h|^{4} \frac{T^{2}}{\left(m_{F}^{2}-m_{\mathrm{dm}}^{2}\right)^{2}},
$$

for an $F$ fermion exchange, unless the $\psi$ and $F$ fields are degenerate in mass, in which case the low-energy transfer scattering cross section gets the usual Thomson behavior

$$
\sigma_{\mathrm{dm}-\nu} \sim \frac{|h|^{4}}{m_{\mathrm{dm}}^{2}}, \quad m_{F}=m_{\mathrm{dm}} .
$$

Similarly, for a $U$ coupling we have

$$
\left\langle\sigma_{\mathrm{dm}-\nu}|v|\right\rangle \sim g_{\psi}^{2} g_{\nu}^{2} \frac{T^{2}}{m_{U}^{4}} .
$$

The Lagrangian density, Eq. (1), also describes the interaction of (chiral) fermionic dark matter with neutrinos via the exchange of a massive scalar field, with the obvious redefinition $\psi \leftrightarrow F$, and scattering cross sections are again given in this case by Eqs. (3) or (4).

Finally, interaction of a Dirac DM field with neutrinos via a vector-boson interaction Lagrangian,

$$
\begin{aligned}
\mathcal{L}_{\text {int }}= & g_{\psi}\left(c_{L} \bar{\psi}_{L} \gamma^{\mu} \psi_{L}+c_{R} \bar{\psi}_{R} \gamma^{\mu} \psi_{R}\right) U_{\mu} \\
& +g_{\nu} \bar{\nu}_{L} \gamma^{\mu} \nu_{L} U_{\mu},
\end{aligned}
$$

gives, for $m_{\mathrm{dm}} \geq T$,

$$
\left\langle\sigma_{\mathrm{dm}-\nu}|v|\right\rangle \sim g_{\psi}^{2} g_{\nu}^{2}\left(c_{L}^{2}+c_{R}^{2}-c_{L} c_{R}\right) \frac{T^{2}}{m_{U}^{4}} .
$$

A light $U$ boson with an order $\mathrm{MeV}$ mass coupled to charged leptons might affect the electron-neutrino scattering cross section at low energy, while measurements show no significant deviations from the standard electroweakmodel result [31,32] (see also Ref. [21] for a detailed analysis on this issue). A possible way out is of course to suppress the value of the $U$ coupling to ordinary matter, including neutrinos, with respect to its coupling to DM particles. In this case, however, neutrino-dark- matter scattering would be quite small and again no observable effects on LSS can be obtained. Another possibility, though less appealing, is to assume that the $U$ boson couples mainly to neutrinos and very weakly to charged leptons.

Regardless of the particular nature of DM particles and the particular coupling to neutrinos, provided their mass as well as the mass of the exchanged particle is in the range of $\mathrm{MeV}$ or larger, we see from our discussion that the typical thermally averaged scattering cross section with neutrinos for $T \leq \mathrm{MeV}$ has two possible distinct behaviors, either decreasing as $T^{2}$ or constant for mass degeneracy of DM and intermediate scalar/fermion particle $F$. It is useful to define the DM-neutrino opacity, the thermally averaged scattering cross section over DM mass ratio. As we will see in the next section, this parameter directly enters the Euler equation describing the neutrino and DM velocity perturbations, since the effect of DM-neutrino scattering is via the product $n_{\mathrm{dm}} \sigma_{\mathrm{dm}-\nu}=\rho_{\mathrm{dm}}\left(\sigma_{\mathrm{dm}-\nu} / m_{\mathrm{dm}}\right)$ with $n_{\mathrm{dm}}$ and $\rho_{\mathrm{dm}}$ the DM number and energy density, respectively. In particular, for the $T^{2}$ behavior as in Eqs. (3) and (5), or (7) we define

$$
\frac{\left\langle\sigma_{\mathrm{dm}-\nu}|v|\right\rangle}{m_{\mathrm{dm}}} \equiv Q_{2} \frac{1}{a^{2}} .
$$

Here, $a$ denotes the scale factor, normalized to unity at the present time. The value of $Q_{2}$ contains all information about the DM-neutrino scattering model, such as coupling constant and the mass scale of DM as well as the exchanged intermediate particle, once the neutrino energy dependence of the cross section, in this case $T^{2}=1 / a^{2}$, has been factorized.

The opacity can be written in terms of the DM-neutrino coupling, which will be generically denoted by $g$ and a mass scale $M$ of order $\mathrm{MeV}$ or larger. For example, for a scalar DM particle coupled to neutrinos via the exchange of a fermion particle $F$ we have $g=|h|$ and $M^{2}=\mid m_{F}^{2}-$ $m_{\mathrm{dm}}^{2}$ l [see Eq. (3)], while for a $U$ exchange, $g^{2}=g_{\psi} g_{\nu}$ and $M=m_{U}$ [see Eq. (5)]. In this notation, and using the known value of neutrino temperature today, we get

$$
Q_{2} \sim \frac{g^{4}}{(M / \mathrm{MeV})^{4}} \frac{1}{m_{\mathrm{dm}} / \mathrm{MeV}} \times 10^{-41} \mathrm{~cm}^{2} \mathrm{MeV}^{-1} .
$$

Similarly, for the case of a constant scattering cross section, as in Eq. (4) we define

$$
\frac{\left\langle\sigma_{\mathrm{dm}-\nu}|v|\right\rangle}{m_{\mathrm{dm}}} \equiv Q_{0},
$$

which gives

$$
Q_{0} \sim \frac{|h|^{4}}{\left(m_{\mathrm{dm}} / \mathrm{MeV}\right)^{3}} \times 10^{-22} \mathrm{~cm}^{2} \mathrm{MeV}^{-1} .
$$

In this case too the value of $Q_{0}$ contains all information about the DM-neutrino scattering model for the case $m_{\mathrm{dm}}=m_{F}$. Upper bounds on these parameters will be discussed in the following section using the LSS power spectrum as well as other astrophysical constraints.

We now come back to the issue of the relic abundance of DM particles $\psi$. In the usual scenario, the present value of the DM energy density results from the freeze-out of annihilation processes at temperatures of the order of $m_{\mathrm{dm}} / 20$; see, e.g., Ref. [33]. As we will see in the next section, the key parameter entering the Euler equation ruling the DM fluid perturbation is the effective DM scattering rate off the neutrino background, defined by

$$
\Gamma_{\mathrm{sc}}=\frac{4}{3} \frac{\rho_{\nu}}{\rho_{\mathrm{dm}}} n_{\mathrm{dm}} \sigma_{d m-\nu},
$$

with $\rho_{\nu}$ the neutrino energy density and we recall that $n_{\mathrm{dm}}$ and $\rho_{\mathrm{dm}}$ stand for the number and energy density of DM particles, respectively. This expression is as usual obtained 
using momentum conservation in scattering processes, which states (see e.g. [11,34]):

$$
\left(\rho_{\nu}+p_{\nu}\right) \Gamma_{\mathrm{sc}}^{(\nu)}>=\left(\rho_{\mathrm{dm}}+p_{\mathrm{dm}}\right) \Gamma_{\mathrm{sc}} \sim \rho_{\mathrm{dm}} \Gamma_{\mathrm{sc}},
$$

$p_{\nu}=\rho_{\nu} / 3$, and $p_{\mathrm{dm}} \ll \rho_{\mathrm{dm}}$ are the neutrino and DM pressure, respectively, and $\Gamma_{\mathrm{sc}}^{(\nu)}$ is the scattering rate of a neutrino off the DM particle background

$$
\Gamma_{\mathrm{sc}}^{(\nu)}>=n_{\mathrm{dm}} \sigma_{\mathrm{dm}-\nu} .
$$

If we assume that $m_{\mathrm{dm}} \geq 10 \mathrm{MeV}$ and that scattering processes with neutrinos are still effective for small temperatures $T \ll \mathrm{MeV}$, thus leaving an imprint on the LSS power spectrum and $\mathrm{CMB}$, annihilation to neutrinos would reduce the DM energy density to a very tiny value today.

To show this let us assume that indeed the annihilation processes $\psi \bar{\psi} \rightarrow \nu \bar{\nu}$ freeze out at temperature of the order of $T_{f}=m_{\mathrm{dm}} / 20 .{ }^{1}$ We first consider the case of a (nonselfconjugated) scalar-DM field. For the coupling to neutrinos via the exchange of a massive fermion $F$ [see Eq. (1)], we get for the annihilation cross section ${ }^{2}$

$$
\sigma_{\mathrm{ann}} \sim|h|^{4} \frac{m_{\mathrm{dm}}^{2}}{\left(m_{F}^{2}+m_{\mathrm{dm}}^{2}\right)^{2}} \frac{T}{m_{\mathrm{dm}}} .
$$

Notice that the $s$-wave annihilation contribution is suppressed by the square of neutrino mass, and has been neglected. This is a consequence of the fact that the coupling is chiral; see, e.g., Ref. [9]. Using Eq. (3), we see that at $T_{f}$ the ratio of the thermally averaged neutrino scattering rate over the annihilation rate $\Gamma_{\mathrm{ann}}=\sigma_{\mathrm{ann}}|v| n_{\mathrm{dm}}$ is of the order of

$$
\frac{\Gamma_{\mathrm{sc}}}{\Gamma_{\mathrm{ann}}} \sim \frac{\left(m_{F}^{2}+m_{\mathrm{dm}}^{2}\right)^{2}}{\left(m_{F}^{2}-m_{\mathrm{dm}}^{2}\right)^{2}} \frac{T_{f}}{m_{\mathrm{dm}}},
$$

when $m_{F} \neq m_{\mathrm{dm}}$ [see Eq. (3)] or

$$
\frac{\Gamma_{\mathrm{sc}}}{\Gamma_{\mathrm{ann}}} \sim 4 \frac{m_{\mathrm{dm}}}{T_{f}},
$$

when $m_{F}=m_{\mathrm{dm}}$ [see Eq. (4)]. This means that if annihilation freezes out at $T \sim m_{\mathrm{dm}}$, neutrino scatterings on DM particles are also largely ineffective at a temperature smaller than $\mathrm{MeV}$. In this case, no signatures of neutrinoDM interactions can be constrained by present observations. The largest scale where DM-neutrino interactions can leave an imprint corresponds to a wave number

$$
k \sim \frac{2 \pi}{c} \frac{H\left(z_{\mathrm{sc}}\right)}{1+z_{\mathrm{sc}}} \sim \frac{1+z_{\mathrm{sc}}}{\sqrt{1+z_{\mathrm{eq}}}} \times 10^{-3} h \mathrm{Mpc}^{-1},
$$

\footnotetext{
${ }^{1}$ In general, DM particles are coupled to the electromagnetic plasma as well and annihilate into $e^{+} e^{-}$pairs. In this case, the corresponding annihilations will be assumed to freeze out at $T_{f}$ or earlier.

${ }^{2}$ For a detailed analysis of scalar-DM annihilation cross section see e.g. the appendix in [21].
}

where $H\left(z_{\mathrm{sc}}\right)$ is the Hubble parameter at the redshift $z_{\mathrm{sc}}$ where DM- $\nu$ interactions freeze out and $z_{\mathrm{eq}}$ is the redshift of matter-radiation equality. For $z_{\mathrm{sc}} \sim 10^{8}$, corresponding to $T \sim 0.01 \mathrm{MeV}$, we obtain $k \sim 10^{3} h \mathrm{Mpc}^{-1}$.

Let us now consider the case of a $U$-exchange interaction. The right order of magnitude ( $10 \mathrm{pb})$ of the ( $p$-wave) annihilation cross section requires a light $U$ boson mass, of the order of $\mathrm{MeV}$, and coupling $h \sim 10^{-3}$. For $T \leq m_{\mathrm{dm}}$, we get

$$
\sigma_{\mathrm{ann}} \sim g_{\psi}^{2} g_{\nu}^{2} \frac{m_{\mathrm{dm}}^{2}}{\left(m_{U}^{2}-4 m_{\mathrm{dm}}^{2}\right)^{2}} \frac{T}{m_{\mathrm{dm}}} .
$$

Comparing this result with Eq. (5), we get at $T_{f}$,

$$
\frac{\Gamma_{\mathrm{sc}}}{\Gamma_{\mathrm{ann}}} \sim \frac{\left(m_{U}^{2}-4 m_{\mathrm{dm}}^{2}\right)^{2}}{m_{U}^{4}} \frac{T_{f}}{m_{\mathrm{dm}}} .
$$

Again neutrino scatterings freeze out quite early around $T_{f}$.

For spin-1/2 DM (Dirac or Majorana) particles and $F$ or $U$ couplings of Eqs. (1) and (6), one can reason along the same lines, with similar results.

The case of a neutral scalar DM particle $\psi$ is quite different. In this case in fact, the $F$ coupling to neutrinos corresponds to an annihilation cross section which vanishes in the limit of massless neutrinos. On the other hand, couplings of DM to charged leptons can produce the correct relic abundance if $m_{F}$ is of the order of $100 \mathrm{GeV}$ to $1 \mathrm{TeV}$ [21]. However, for such large values of $m_{F}$, the scattering cross section is very small too (in particular it vanishes in the local limit $m_{F} \rightarrow \infty$ due to a cancellation between the $s$ - and $u$-channel amplitudes), so the case of self-conjugated scalar-DM-neutrino coupling is of no interest for the purposes of this paper, and is not expected to produce any observable features in the LSS.

Summarizing, if we assume that DM couples to neutrinos strongly enough to produce observable effects that can be constrained by CMB and LSS observations, we have to abandon the idea that relic DM density formed via the usual mechanism based on freezing of DM annihilation processes at temperatures $T \sim m_{\mathrm{dm}}$.

How can our scenario be reconciled with the observed DM contribution to the present energy density of the Universe? First of all, it should be mentioned that only a fraction of the total DM could be coupled to neutrinos. In fact, we will consider this case too in the following. However, this would not represent a solution to the problem, since this neutrino-coupled component would completely annihilate into neutrinos at $T_{f}$. The more interesting possibility is therefore that there is a particle-antiparticle asymmetry produced at higher temperatures in the DM sector coupled to neutrinos, very much like the mechanism by which the baryon (and lepton, in the framework of leptogenesis) number is produced in the early Universe. Indeed, this possibility is also motivated by the intriguing observation that the parameters $\Omega_{b}$ and $\Omega_{\mathrm{dm}}$ only differ by 
a factor five today, yet their production mechanism is usually considered to be quite distinct, with very few exceptions [35,36]; see also the discussion on this point in Ref. [21]. Though a more detailed theoretical analysis of this possibility is perhaps still needed, we think that this idea still represents a stimulating possible scenario. In this case, it is meaningful to check to what extent the present data can constrain strong couplings of DM particles with mass in the range $\mathrm{MeV}$ or higher to neutrinos. This scenario requires that DM particles are excitations of a nonselfconjugated field, such as a complex scalar field or a Dirac field, and that a particle-antiparticle asymmetry in the DM sector $\eta_{\psi}=\left(n_{\psi}-n_{\bar{\psi}}\right) / n_{\gamma}$ has been produced at some early stage, of the order of

$$
\eta_{\psi}=\eta_{B} \frac{\Omega_{\mathrm{dm}}}{\Omega_{b}} \frac{m_{p}}{m_{\mathrm{dm}}}
$$

with $m_{p}$ the proton mass and $\eta_{B}$ the baryon-to-photon ratio, $\eta_{B} \sim 6.3 \times 10^{-10}$; see e.g., Ref. [29]. The analysis performed in the following sections relies on these assumptions.

We conclude this section by reporting the expression of the smaller-wave mode $k$ for which we expect to see the effects of DM- $\nu$ scatterings in the dark-matter perturbation in terms of the opacities $Q_{2}$ and $Q_{0}$ introduced in Eqs. (9) and (11). This might be useful to understand the results reported in the following. From the definition of $z_{\mathrm{sc}}$,

$$
\Gamma_{\mathrm{sc}}\left(z_{\mathrm{sc}}\right) \sim H\left(z_{\mathrm{sc}}\right),
$$

and using standard values for the neutrino temperature today and assuming no extra relativistic degrees of freedom in addition to photons and neutrinos, it is easy to get from Eq. (18)

$$
k \sim 0.2\left(\frac{10^{-41} \mathrm{~cm}^{2} \mathrm{MeV}^{-1}}{Q_{2}}\right)^{1 / 4} h \mathrm{Mpc}^{-1},
$$

or, for a constant scattering cross section,

$$
k \sim 0.2 \times 10^{-5}\left(\frac{10^{-22} \mathrm{~cm}^{2} \mathrm{MeV}^{-1}}{Q_{0}}\right)^{1 / 2} h \mathrm{Mpc}^{-1} .
$$

\section{NIDM AND STRUCTURE FORMATION}

In order to include a neutrino-dark-matter interaction, we modify the standard Euler equations resulting in (using conformal time)

$$
\begin{gathered}
\dot{\theta}_{\mathrm{dm}}=-\frac{\dot{a}}{a} \theta_{\mathrm{dm}}+\frac{4 \rho_{\nu}}{3 \rho_{\mathrm{dm}}} a n_{\mathrm{dm}} \sigma_{\mathrm{dm}-\nu}\left(\theta_{\nu}-\theta_{\mathrm{dm}}\right), \\
\dot{\theta}_{\nu}=k^{2}\left(\frac{1}{4} \delta_{\nu}-\sigma_{\nu}\right)+a n_{\mathrm{dm}} \sigma_{\mathrm{dm}-\nu}\left(\theta_{\mathrm{dm}}-\theta_{\nu}\right),
\end{gathered}
$$

where momentum conservation in scattering processes has been accounted for. With $\theta$ we denote the velocity perturbations, the subscripts " $\mathrm{dm}$ " and " $\nu$ " standing for neutrino-interacting dark matter and neutrinos, respec- tively. The quantity $a n_{\mathrm{dm}} \sigma_{\mathrm{dm}-\nu}$ is the differential opacity and gives as mentioned already the scattering rate of neutrinos by dark matter.

As in the case of the baryon-photon interaction, we neglect both the shear term $k^{2} \sigma$ and the term $c_{\mathrm{dm}}^{2} k^{2} \delta$, where $c_{\mathrm{dm}}$ is the sound speed of the dark-matter fluid.

As seen in the previous section, the coupling between neutrinos and dark matter can be parametrized through a cross section that either decreases as $a^{-2}$, or takes a constant value. In these two cases, the parameters determining the DM perturbations are the opacities (cross section to DM-mass ratios) $Q_{2}$ and $Q_{0}$ defined in Eqs. (8) and (10).

First consider the case $\left\langle\sigma_{\mathrm{dm}-\nu}|v|\right\rangle \propto a^{-2}$ (similar considerations can be made for the constant cross section). In Fig. 1, we show what happens when a perturbation of wave number $k=1.04 h \mathrm{Mpc}^{-1}$ enters the horizon for different values of $Q_{2}$. If the coupling is zero, we have the standard picture. The mode enters the horizon in the radiationdominated era, and it starts to grow first logarithmically and then linearly with the expansion factor (during matter domination). When the same mode enters the horizon with $Q_{2}=5 \times 10^{-44} \mathrm{~cm}^{2} \mathrm{MeV}^{-1}$, the growth is nearly zero during the radiation epoch, while the mode starts growing linearly with the scale factor during matter domination, since the coupling with neutrinos becomes negligible in this stage for the chosen value of $Q_{2}$.

The situation is different when we consider a stronger coupling, say $Q_{2}=10^{-39} \mathrm{~cm}^{2} \mathrm{MeV}^{-1}$. When the perturbation enters the horizon, dark matter is coupled with neutrinos and this results in a series of oscillations until decoupling is reached. Notice that the amplitude of oscillations decreases near decoupling, because the decoupling itself is not instantaneous and so we see diffusion damping for the dark-matter-neutrino fluid. In Fig. 2, we plot several

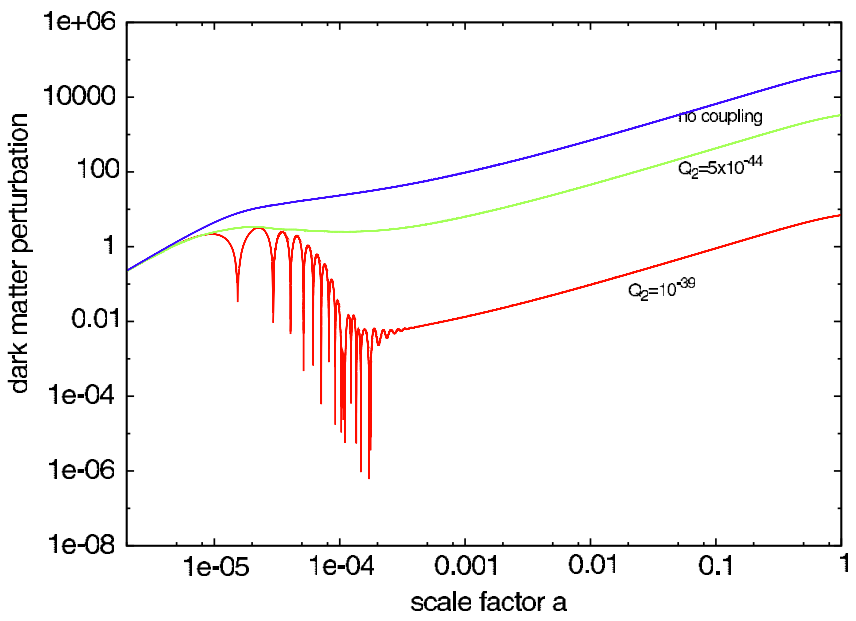

FIG. 1 (color online). Dark-matter perturbations of $k=$ $1.04 \mathrm{~h} \mathrm{Mpc}^{-1}$; the opacity $Q_{2}$ is in the unit of $\mathrm{cm}^{2} \mathrm{MeV}^{-1}$. Damped oscillations are clearly seen for $Q_{2}=$ $10^{-39} \mathrm{~cm}^{2} \mathrm{MeV}^{-1}$. 

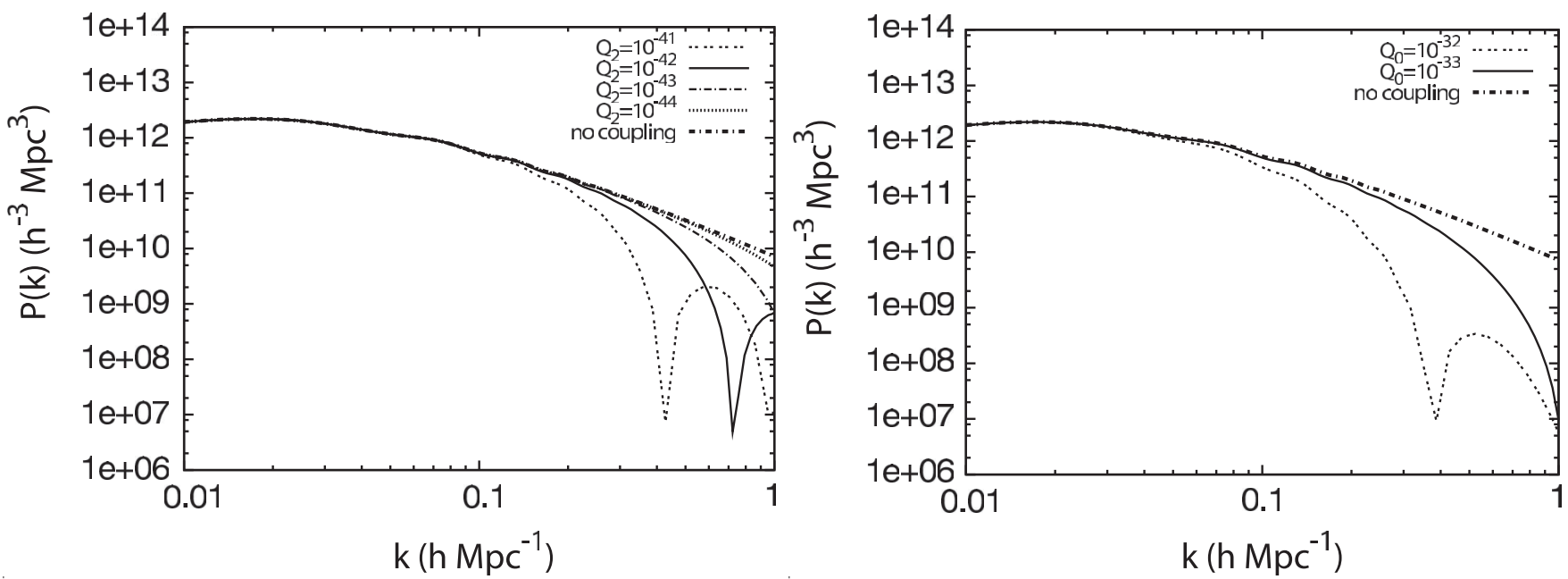

FIG. 2. Several matter power spectra with different opacities $Q_{2}$ (top panel) and $Q_{0}$ (bottom panel) between dark matter and neutrinos; $Q_{2}$ and $Q_{0}$ are in units of $\mathrm{cm}^{2} \mathrm{MeV}^{-1}$.

matter power spectra for different values of the dark-matter-neutrino interaction for both the couplings considered. The effect of the dark-matter-neutrino interaction can be seen on small scales in the matter power spectrum. Larger couplings will correspond to later epochs of neutrino-DM decoupling and to a damped oscillating regime on larger scales. Finally, in Fig. 3, we plot the angular power spectra of CMB anisotropies for two models with and without DMneutrino coupling. For the value of $Q_{2}$ we consider, which is already at odds with current clustering data, there is a small enhancement in the small-scale CMB anisotropies. The reason for this is that the anisotropic stress in the neutrino relativistic component is reduced due to the coupling. In other words, neutrinos are no more a fluid with a "viscosity parameter" $c_{\text {vis }}^{2}=1 / 3$. This parameter, introduced in Ref. [37], controls the relationship between velocity/metric shear and anisotropic stresses in the neutrino

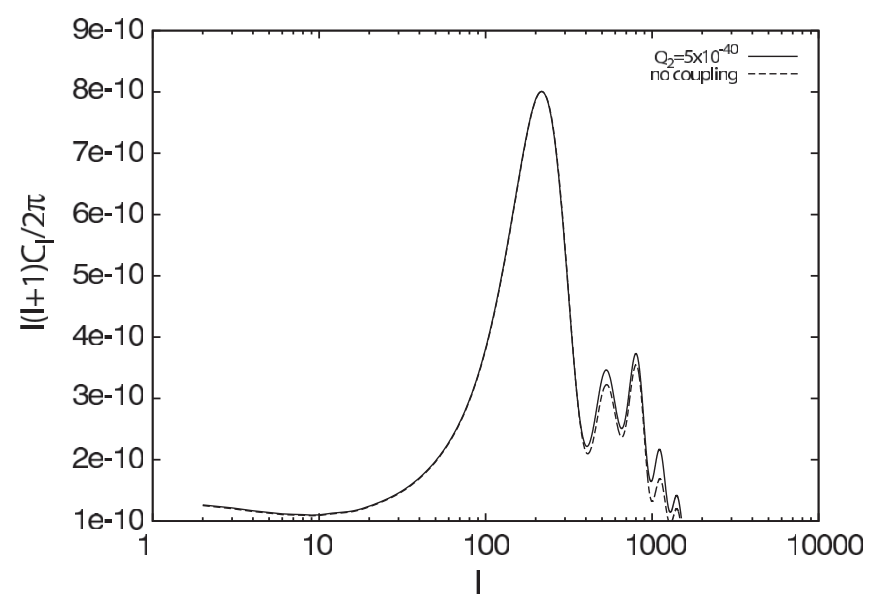

FIG. 3. Angular power spectra with and without dark-matterneutrino coupling. A small enhancement $(\sim 10 \%)$ of the height of the peaks on small scales is observed. background [38]. The value of $c_{\mathrm{vis}}^{2}$ will be close to $c_{\mathrm{vis}}^{2}=0$ and this implies a small enhancement of the small-scale peaks, at the level of $10 \%$ [38]. In order to bound the strength of DM coupling to neutrinos, we consider the real-space power spectrum of galaxies in the Sloan Digital Sky Survey (SDSS) using the data and window functions of the analysis of Ref. [39]. To compute the likelihood function $\mathcal{L}^{\text {SDSS }}$ for the SDSS, we restrict the analysis to a range of scales over which the fluctuations are assumed to be in the linear regime $\left(k<0.2 h^{-1} \mathrm{Mpc}\right)$, and we marginalize over a bias $b$ considered to be an additional free parameter. Since no relevant signature is expected on CMB anisotropies for the values of $Q_{2}$ and $Q_{0}$ we consider, we do not include CMB-anisotropy data in the analysis and assume a cosmological concordance model with $\Omega_{\Lambda}=$ 0.70 and $\Omega_{\mathrm{dm}}=0.25$, which produces a good fit to current CMB data.

By evaluating the SDSS likelihood we found that the couplings are constrained to be

$$
\begin{aligned}
& Q_{2} \leq 10^{-42} \mathrm{~cm}^{2} \mathrm{MeV}^{-1}, \\
& Q_{0} \leq 10^{-34} \mathrm{~cm}^{2} \mathrm{MeV}^{-1},
\end{aligned}
$$

at the $2 \sigma$ confidence level in above fiducial cosmology. If we compare Eq. (27) with Eq. (9), we see that for couplings $g$ of order one this bound is saturated if both $m_{\mathrm{dm}}$ and $M$ are of the order of MeV. Smaller values of $g$ imply lighter masses for the NIDM and the intermediate exchanged particle in the scattering process. In view of the $\mathrm{BBN}$ bound mentioned in Sec. II, $m_{\mathrm{dm}} \geq 10 \mathrm{MeV}$, these values are already disfavored, so the LSS constraint we obtain is not further constraining the NIDM scenario. In fact, the $\mathrm{BBN}$ can be weakened if we allow for more exotic features in the neutrino density, in particular, a neutrino chemical potential. Indeed, in this case, the larger contribution of DM particles to the Hubble expansion rate resulting into a 
higher ${ }^{4} \mathrm{He}$ mass fraction can be compensated by a positive (i.e., more neutrinos than antineutrinos) value of the chemical potential; see, e.g., Ref. [27]. Further bounds might be obtained by studying the LSS power spectrum at larger wave numbers, $k \geq 0.2 h \mathrm{Mpc}^{-1}$, taking into account the nonlinear behavior of perturbations for very small scales.

On the other hand, the result for $Q_{0}$ which we recall corresponds to an intermediate particle and NIDM mass degeneracy, is more severely constraining $m_{\mathrm{dm}}$. We get in this case $m_{\mathrm{dm}} \geq 10 h^{4 / 3} \mathrm{GeV}$.

\section{ASTROPHYSICAL CONSTRAINTS}

It is interesting to compare Eqs. (27) and (28) with the bounds on $Q_{2}$ and $Q_{0}$ that can be obtained from the propagation of astrophysical neutrinos. The most important constraint is provided by observation of neutrinos from SN1987A [40], which are in good agreement with the theoretical expectation of neutrino fluxes from type II supernovae. These neutrinos have energies of order $10 \mathrm{MeV}$. The thickness of the dark-matter layer that they propagate through is approximately $\int \rho(l) d l$, the integral of the dark-matter density along the line of sight $l$ to the large magellanic cloud (LMC). Approximating the darkmatter density $\rho(l) \sim \rho_{0}\left(l / l_{0}\right)^{-2}$, where $\rho_{0} \simeq$ $0.4 \mathrm{GeV} \mathrm{cm}^{-3}$ is the local density and $l_{0} \simeq 8 \mathrm{kpc}$ our distance from the galactic center, we find a dark-matter thickness $\sim 10^{25} \mathrm{MeV} \mathrm{cm}^{-2}$. Given the agreement between the predicted and observed neutrino flux and energy spectrum, we infer that neutrinos from SN1987A were not significantly absorbed by dark matter along the line of sight, from which we get an upper bound $\sim 10^{-25} \mathrm{~cm}^{2} \mathrm{MeV}^{-1}$ to the neutrino-DM opacity for neutrinos of energy $\sim 10 \mathrm{MeV}$. From this result we obtain the

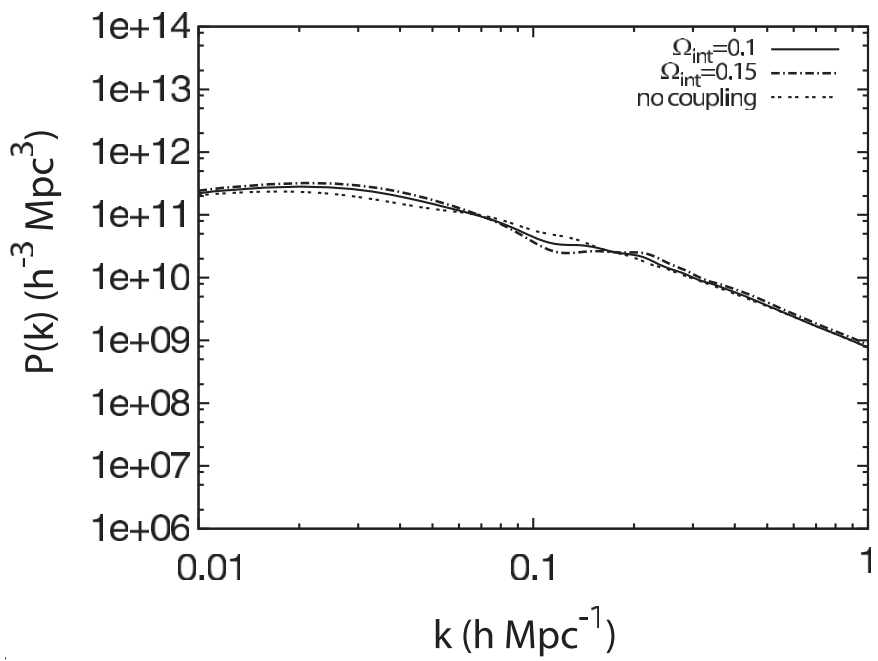

upper bounds

$$
\begin{gathered}
Q_{2} \leq 10^{-47}\left(\frac{10 \mathrm{MeV}}{M}\right)^{2} \mathrm{~cm}^{2} \mathrm{MeV}^{-1}, \\
Q_{0} \leq 10^{-25} \mathrm{~cm}^{2} \mathrm{MeV}^{-1} .
\end{gathered}
$$

We note that the bound on $Q_{2}$ is stronger than what is obtained using LSS data and of the same order of magnitude as the BBN limit corresponding to $M \geq 10 \mathrm{MeV}$, while for $Q_{0}$ the stronger bound is still provided by Eq. (28).

Neutrinos with high energy are likely to be produced by a variety of astrophysical sources. Strong scattering of these neutrinos off the NIDM when traveling over cosmological distances of order of tens of Mpc implies large energy losses and correspondingly a strong deformation of the emitted energy spectrum at the source. For light NIDM and intermediate exchanged particles (in the $10 \mathrm{MeV}$ range) the high-energy $\left(E_{\nu} \geq \mathrm{GeV}\right)$ scattering cross section behaves as $\sigma_{\mathrm{dm}-\nu}\left(E_{\nu} \gg 10 \mathrm{MeV}\right) \sim g^{4} / s$ with $s=m_{\mathrm{dm}} E_{\nu}$. We stress once more that high values for NIDM or intermediate $F$ or $U$ particle mass, though perfectly legitimate, implies no observable effects in the LSS power spectrum and are thus of no interest for the present analysis. Using the definition of $Q_{2}$ and $Q_{0}$ of Sec. II and the value of the critical density today, we can evaluate the typical scattering length as a function of the neutrino energy as follows

$$
\begin{aligned}
& \frac{\lambda_{\nu}}{10 \mathrm{Mpc}} \sim \frac{E_{\nu}}{M} \frac{1}{\Omega_{\mathrm{dm}}} \frac{1}{Q_{2}} 10^{-44} \mathrm{~cm}^{2} \mathrm{MeV}^{-1}, \\
& \frac{\lambda_{\nu}}{10 \mathrm{Mpc}} \sim \frac{E_{\nu}}{M} \frac{1}{\Omega_{\mathrm{dm}}} \frac{1}{Q_{0}} 10^{-24} \mathrm{~cm}^{2} \mathrm{MeV}^{-1} .
\end{aligned}
$$

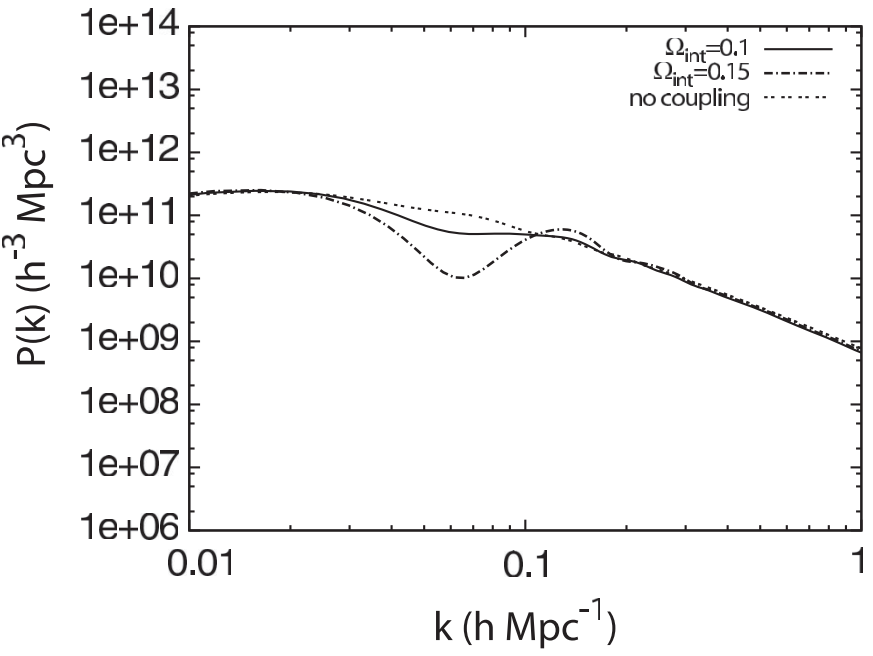

FIG. 4. Matter power spectra for cold + interacting dark matter with $Q_{2}=10^{-38} \mathrm{~cm}^{2} \mathrm{MeV}^{-1}$ (top panel) and $Q_{2}=$ $10^{-37} \mathrm{~cm}^{2} \mathrm{MeV}^{-1}$ (bottom panel). 
If we use the LSS bounds of Eqs. (27) and (28) we see that for $M \sim 10 \mathrm{MeV}$, the value of $\lambda_{\nu}$ is typically very large. The effect of interactions with NIDM can only affect neutrinos with order-GeV energy over distances of $10 \div$ $100 \mathrm{Mpc}$ assuming the largest value for $Q_{2}$, while the effect is negligible for higher values of $E_{\nu}$ or for the $Q_{0}$ case.

The bounds discussed so far are obtained under the assumption that all dark matter is interacting with neutrinos. However, if the dark matter is made of several components, it is possible that only a fraction of the dark matter was actually strongly coupled. In Fig. 4, we show matter power spectra for a standard $\Lambda \mathrm{CDM}$ model with $\Omega_{\mathrm{dm}}=$ 0.25 and $\Omega_{\Lambda}=0.7$ and for other models where a fraction of the energy density $\Omega_{\text {int }}=0.7-\Omega_{\Lambda}$ of the cosmological constant is replaced by interacting dark matter with coupling $Q_{2}$. As we can see, the spectra are quite similar if we consider quite large values of $Q_{2}$, as large as $10^{-38} \mathrm{~cm}^{2} \mathrm{MeV}^{-1}$. This is simply due to the fact that the interacting component is nearly unclustered on large scales, $k \sim 0.01 h \mathrm{Mpc}^{-1}$. Therefore, adding this component or changing the energy density in $\Lambda$ is nearly equivalent. This degeneracy in the framework of NIDM might weaken the current estimates of the matter density from galaxy clustering. For higher values of $Q_{2}$ or $\Omega_{\text {int }}$, oscillations in the power spectrum are instead clearly visible.

In Fig. 5, we plot constraints on $Q_{2}$ using $\operatorname{SDSS} P(k)$ data by allowing this possibility that only a fraction of the dark matter interacts with neutrinos. The overall matter density is fixed at $\Omega_{m}=0.27$ and we assume a flat universe. As we can see, a smaller $\Omega_{\mathrm{NIDM}}$ allows the possibility of relaxing the constraints on $Q_{2}$. Recently, experimental evidence for a "peak" in the correlation function of the SDSS luminous red galaxy (LRG) distribution at $100 \mathrm{Mpc}$ scales has been reported [41]. This peak is generally interpreted as the imprint of oscillations in the

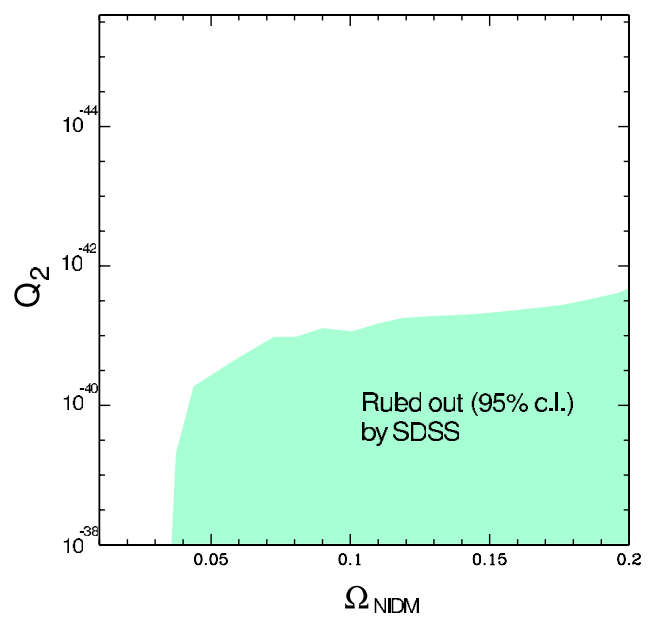

FIG. 5 (color online). Constraints on the $Q_{2}$ vs $\Omega_{\text {NIDM }}$ plane from SDSS $P(k)$ measurements. An overall matter density of $\Omega_{m}=0.27$ is assumed with $\Omega_{b}=0.04$.

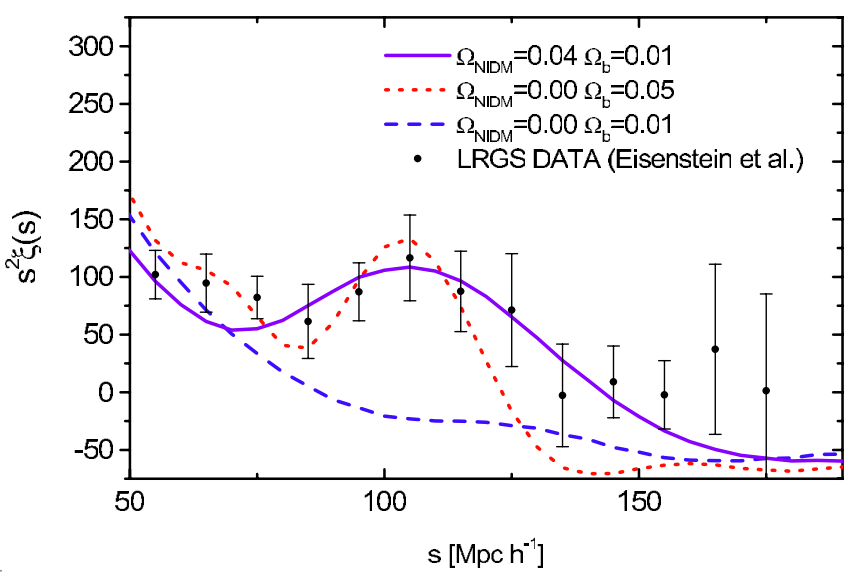

FIG. 6 (color online). Degeneracy for the baryon acoustic peak in the galaxy correlation function. Three models are plotted: (1) a standard CDM model with a baryon density that reproduces the baryon peak (dotted line), (2) a standard CDM model with a low baryon density (dashed line), and (3) a NIDM model with a low baryon density (solid line). While a CDM model with a low baryon density fails to describe the peak, a NIDM model with a low baryon density describes the peak adequately. The data are taken from the LRG analysis of Eisenstein et al. 2005.

photon-baryon fluid near recombination. In the standard CDM framework, this peak serves as an absolute ruler and with clustering measurements as a function of redshift, one can extract strong constraints on the dark-energy time evolution. However, it is clear that if a portion of the dark matter is oscillating as a consequence of strong coupling with neutrinos, that component may also produce an oscillatory behavior and affect the conclusions of Ref. [41]. In order to show this effect more quantitatively we compare the LRG data in Fig. 6 with the correlation function computed under three model descriptions: a standard CDM model with a baryon density that reproduces the baryon peak, a combination of a standard CDM model with a low baryon density, and a model with a NIDM component and a low baryon density. ${ }^{3}$

As we can see, NIDM is able to mimic the baryonic peak even in the case of a low baryon density universe though in the standard CDM, a low baryon density will not describe the oscillation. While the possibility shown in Fig. 6 is certainly fine tuned, one may need to allow for such a scenario when deriving constraints on the baryon energy density $\Omega_{b} h^{2}$ from large-scale-structure observations if departures from the standard description were to be considered. Let us note, however, that the above scenario, while mimicking baryonic oscillations in galaxy clustering with NIDM, would lead to a different shape for the CMB-

\footnotetext{
${ }^{3}$ As suggested in Ref. [41], using $N$-body simulations, to compare between measurements in the redshift space and predictions in the real space, we multiply the predicted correlation functions by a conversion factor of $\left[1+0.06 /\left(1+(0.06 s)^{6}\right)\right]^{2}$, where $s$ is the physical scale measured in $h^{-1} \mathrm{Mpc}$.
} 
anisotropy power spectrum. A combined analysis is therefore a powerful tool for detecting NIDM in mixed models and any discrepancy between the value of the baryon density derived independently from those data sets could hint for a NIDM component.

\section{DISCUSSION AND CONCLUSIONS}

In this paper, we have studied the cosmological consequences of a possible coupling between neutrinos and light dark matter with mass in the $\mathrm{MeV}$ range. We considered two possible behaviors for the thermally averaged neutrino-DM elastic-scattering cross section, either decreasing with temperature as $T^{2}$ or constant. We compared the NIDM scenario with the large-scale galaxy distribution and obtained upper limits on the opacity (ratio of the DMneutrino cross section to the dark-matter mass) of $Q_{2}<$ $10^{-42} \mathrm{~cm}^{2} \mathrm{MeV}^{-1}$ and $Q_{0}<10^{-34} \mathrm{~cm}^{2} \mathrm{MeV}^{-1}$ at the $95 \%$ C.L. These limits may be relaxed if one considers the possibility that only a fraction of the dark matter is made of NIDM. The main cosmological observable for NIDM consists in diffusion-damped oscillations in the matter power spectrum. Those NIDM oscillations may affect current cosmological constraints on neutrinos masses and dark energy from galaxy clustering. We have stressed that strongly coupled DM particles would have a nonnegligible relic abundance today only if an asymmetry between DM particle and antiparticle is produced at some early stage in the evolution of the Universe, since their density would vanish today because of effective annihilation processes into neutrinos down to temperatures much smaller than the DM mass. Detection of NIDMinduced oscillations in the LSS power spectrum would be a hint for such a nonstandard scenario.

\section{ACKNOWLEDGMENTS}

We acknowledge the use of the publicly available numerical code CMBFAST. G. M. is pleased to thank P.D. Serpico for discussions. A. M. is supported by MURST through COFIN Contract No. 2004027755 and by ALFAEC funds in the framework of Program HELEN (High Energy Physics Latinoamerican-European Network). M. K. is supported by DOE No. DE-FG03-92-ER40701, NASA No. NNG05GF69G, and the Gordon and Betty Moore Foundation.
[1] G. Jungman, M. Kamionkowski, and K. Griest, Phys. Rep. 267, 195 (1996); L. Bergstrom, Rep. Prog. Phys. 63, 793 (2000); G. Bertone, D. Hooper, and J. Silk, Phys. Rep. 405, 279 (2005).

[2] M.S. Turner, Phys. Rep. 197, 67 (1990); G. G. Raffelt, Phys. Rep. 198, 1 (1990); L. J. Rosenberg and K. A. van Bibber, Phys. Rep. 325, 1 (2000).

[3] R. Schaeffer and J. Silk, Astrophys. J. 292, 319 (1985).

[4] A. A. Klypin, A. V. Kravtsov, O. Valenzuela, and F. Prada, Astrophys. J. 522, 82 (1999); B. Moore, S. Ghigna, F. Governato, G. Lake, T. Quinn, J. Stadel, and P. Tozzi, Astrophys. J. 524, L19 (1999).

[5] M. Kamionkowski and A. R. Liddle, Phys. Rev. Lett. 84, 4525 (2000).

[6] R. Schaeffer and J. Silk, Astrophys. J. 332, 1 (1988).

[7] K. Sigurdson and M. Kamionkowski, Phys. Rev. Lett. 92, 171302 (2004).

[8] C. Boehm, A. Riazuelo, S. H. Hansen, and R. Schaeffer, Phys. Rev. D 66, 083505 (2002).

[9] X.1. Chen, M. Kamionkowski, and X.m. Zhang, Phys. Rev. D 64, 021302 (2001).

[10] K. Sigurdson, M. Doran, A. Kurylov, R. R. Caldwell, and M. Kamionkowski, Phys. Rev. D 70, 083501 (2004); 73, 089903 (2006).

[11] C. Boehm and R. Shaeffer, Astron. Astrophys. 438, 419 (2005).

[12] S. Profumo, K. Sigurdson, and M. Kamionkowski, Phys. Rev. Lett. 97, 031301 (2006).

[13] D. N. Spergel and P. J. Steinhardt, Phys. Rev. Lett. 84, 3760 (2000).
[14] J.P. Ostriker and P.J. Steinhardt, Science 300, 1909 (2003).

[15] G. Mangano, G. Miele, S. Pastor, T. Pinto, O. Pisanti, and P. D. Serpico, Nucl. Phys. B729, 221 (2005).

[16] S. Hannestad, J. Cosmol. Astropart. Phys. 02 (2005) 011.

[17] S. Hannestad and G. G. Raffelt, Phys. Rev. D 72, 103514 (2005).

[18] N. F. Bell, E. Pierpaoli, and K. Sigurdson, Phys. Rev. D 73, 063523 (2006).

[19] J. F. Beacom, N. F. Bell, and S. Dodelson, Phys. Rev. Lett. 93, 121302 (2004)

[20] C. Boehm, T. A. Ensslin, and J. Silk, J. Phys. G 30, 279 (2004).

[21] C. Boehm and P. Fayet, Nucl. Phys. B683, 219 (2004).

[22] C. Boehm, D. Hooper, J. Silk, and M. Casse, Phys. Rev. Lett. 92, 101301 (2004).

[23] D. Hooper, F. Ferrer, C. Boehm, J. Silk, J. Paul, N. W. Evans, and M. Casse, Phys. Rev. Lett. 93, 161302 (2004).

[24] C. Boehm, P. Fayet, and J. Silk, Phys. Rev. D 69, 101302 (2004).

[25] P. D. Serpico and G. G. Raffelt, Phys. Rev. D 70, 043526 (2004).

[26] R. H. Cyburt, B. D. Fields, and K. A. Olive, Phys. Lett. B 567, 227 (2003).

[27] A. Cuoco, F. Iocco, G. Mangano, G. Miele, O. Pisanti, and P. D. Serpico, Int. J. Mod. Phys. A 19, 4431 (2004).

[28] R. H. Cyburt, B. D. Fields, K. A. Olive, and E. Skillman, Astropart. Phys. 23, 313 (2005).

[29] P. D. Serpico, S. Esposito, F. Iocco, G. Mangano, G. Miele, and O. Pisanti, J. Cosmol. Astropart. Phys. 12 (2004) 010. 
[30] P. Fayet, D. Hooper, and G. Sigl, Phys. Rev. Lett. 96, 211302 (2006).

[31] R. C. Allen et al., Phys. Rev. D 47, 11 (1993).

[32] L. B. Auerbach et al., Phys. Rev. D 63, 112001 (2001).

[33] E.W. Kolb and M.S. Turner, The Early Universe (Addison-Wesley, Redwood City, 1989).

[34] C. P. Ma and E. Bertschinger, Astrophys. J. 455, 7 (1995).

[35] D. B. Kaplan, Phys. Rev. Lett. 68, 741 (1992).

[36] D. Hooper, J. March-Russell, and S. M. West, Phys. Lett. B 605, 228 (2005).
[37] W. Hu, Astrophys. J. 506, 485 (1998).

[38] R. Trotta and A. Melchiorri, Phys. Rev. Lett. 95, 011305 (2005).

[39] M. Tegmark et al. (SDSS Collaboration), Astrophys. J. 606, 702 (2004).

[40] See, e.g., G. G. Raffelt, Stars as Laboratory for Fundamental Physics (University of Chicago, Chicago, 1996).

[41] D. J. Eisenstein et al., Astrophys. J. 633, 560 (2005). 